\title{
Hacia un nuevo modelo de gestión medioambiental
}

\author{
Camilo PRAdo RomÁn \\ camilo.prado.roman@urjc.es \\ Alicia Blanco GonZÁLEZ \\ alicia.blanco@urjc.es \\ Francisco DíEz MARTíN \\ francisco.diez@urjc.es \\ Departamento de Economía de la Empresa. Universidad Rey Juan Carlos
}

Recibido: 1 de Marzo de 2011

Enviado a evaluar: 2 de Marzo de 2011

Aceptado: 5 de Octubre de 2011

\section{RESUMEN}

El estudio del análisis de prospectiva en la gestión medioambiental advierte que la gestión del medio ambiente es utilizada, generada y desarrollada, sólo si respalda los intereses de la organización. Mediante la utilización de modelos estructurales de prospectiva, se ha desarrollado un modelo de futuro para la gestión medioambiental, estableciendo las variables más influyentes. La investigación ha servido para mostrar que los objetivos de actuación directa en los procesos de gestión medioambiental pierden con el tiempo su carácter finalista, pasando a ocupar su lugar los objetivos ligados a la excelencia de gestión, esto es, calidad, ambiente y responsabilidad social.

Palabras clave: Gestión Medioambiental, escenarios futuros

\section{Towards a new model of environmental management}

\begin{abstract}
Prospective analysis brought us closer to the future on the basis of the current reality. This analysis advised us that environmental management is used, generated and developed, only if it supports the interests of the organization. Using prospective structural models, we have developed an environmental management model for the future by establishing the most influential and most dependent variables. Research shows that direct action objectives of environmental management processes lose their finalist character over time, turning it into objectives linked to excellence: quality, environment and social responsibility.
\end{abstract}

Keywords: Environmental Management, Prospective Studies. 


\title{
Vers un nouveau modèle de gestion environnemental
}

\begin{abstract}
RESUME
L'étude de l'analyse de prospective dans la gestion environnementale remarque que la gestion de l'environnement est utilisée, générée et développée, seulement si elle appuie les intérêts de l'organisation. A travers l'utilisation de modèles structurels de prospective, il s'est développé un modèle de future pour la gestion environnementale, établissant les variables les plus influentes. La recherche a servi pour montrer que les objectifs d'action directe sur les procédés de gestion environnementale perdent avec le temps leur caractère final, passant à occuper leur place les objectifs liés a l'excellence de gestion, tel que, qualité, atmosphère et responsabilité sociale.
\end{abstract}

Mots clé: Gestion environnementale, scénarios futurs.

\section{INTRODUCCIÓN}

La respuesta de las organizaciones a las cuestiones medioambientales ha sido objeto de análisis por numerosos investigadores, consiguiendo éxitos parciales pero sin obtener respuestas generalizables ni modelos que permitan visionar el problema de una forma amplia y comprensiva. La búsqueda de los estímulos que llevan a una empresa a aceptar de buen grado dar una respuesta consistente a su entorno natural, acomodando su actividad a los requerimientos legislativos, e incluso ir más lejos en su comportamiento ambiental (Lampe et al, 1991; Lawrence y Morell, 1995; Vredenburg y Westley, 1993; Winn, 1995), ha conseguido delimitar las motivaciones e incluso alguno de los factores que influyen en este tipo de acciones.

Aunque se han realizado estudios sobre los motivos que llevan a las corporaciones a actuar con responsabilidad ambiental y se han conseguido identificar algunas de las motivaciones más importantes (el cumplimiento de la normativa de los gobiernos, la obtención de ventajas competitivas, la presión de los grupos de interés, la ética como principio de actuación, la existencia de acontecimientos críticos que llevan a las organizaciones a replantear su enfoque hacia la responsabilidad con la sociedad o la iniciativa de la dirección superior) los resultados se son limitados a efecto de la predicción de la responsabilidad ambiental (Bansal y Roth, 2000)

No obstante, las cuestiones centrales siguen sin conseguir una respuesta terminante. ¿Por qué algunas empresas adoptan iniciativas que demuestran una alta responsabilidad con su medioambiente, mientras que otras, aparentemente similares en muchos aspectos, incluidas las circunstancias que la rodean, no llegan ni siquiera a cumplir con los mínimos marcados por la legislación?

Aunque son muchos los factores que concurren, al repasar las investigaciones (Bansal y Roth, 2000; Berry y Rondinolli, 1998) que abordan cuestiones ambientales, se encuentra un elemento común, la carencia de estudios longitudinales. El problema de los análisis en relación con el medioambiente es que las organizaciones pueden ser similares en dimensión, internacionalización, equipos directivos o ubicación. Pero esa aparente uniformidad que proporciona tranquilidad al investigador y 
le da coherencia formal al análisis, en ocasiones, produce diferencias en aspectos no fácilmente percibidos.

Las alternativas de las organizaciones respecto a su responsabilidad medioambiental están relacionadas con la excelencia en la gestión (Porter y Van der Linde, 1995). Esta gestión evoluciona, progresa con diversas orientaciones y con intensidades diferentes pero siempre en una línea temporal. No es posible pasar de cero a cien sin asimilar y desarrollar un proceso de gestión que inevitablemente cambia por la maduración que proporciona la experiencia y la cultura que surge en la organización siguiendo la idea de aprender mientras se hace. En consecuencia, organizaciones aparentemente similares pueden tener enormes diferencias en sus condiciones y actitudes para abordar los temas ambientales y en estas diferencias se encuentra el punto hasta al que se ha llegado en el momento del análisis que condiciona sus objetivos y enfoques.

De este modo, el objeto de la investigación actual se encuentra en el cambio en la gestión del medioambiente por las organizaciones. La gestión medioambiental actual no puede orientarse exclusivamente al análisis de la relación actual entre variables, sino que debe abordarse en relación a su evolución. Con este objetivo, se plantea un modelo de gestión medioambiental a medio o largo plazo aplicando una herramienta prospectiva para analizar su evolución. En este caso utilizaremos el análisis de Matriz de Impactos Cruzados - Multiplicación Aplicada a una Clasificación (MICMAC)

En relación con la información disponible en la actualidad, la descripción del sistema de gestión ambiental está relacionada con las percepciones que tienen las organizaciones, y sobre todo sus dirigentes, acerca de su responsabilidad social, obligaciones legales, condiciones económicas, marco de competencia, visión del futuro y otras cuestiones relevantes. Brockhoff et al. (1999) estudia la relevancia de las percepciones centrado en las empresas químicas alemanas y norteamericanas, y confirma diferencias sustanciales en las estrategias empresariales respecto a su gestión ambiental.

Para trabajar con una visión más amplia se necesita disponer de un horizonte donde los escenarios que se dibujan en el futuro se encuentren ligados a la situación actual como consecuencia de la evolución en las relaciones, la importancia de las variables y su dependencia. Un modelo de prospectiva que permita ubicar en un espacio temporal a cualquier organización actual y que oriente acerca del previsible horizonte de las estrategias y comportamientos organizativos.

La dificultad que hay para generar escenarios fundamentados se produce por la naturaleza no-estructurada, compleja, dinámica e incierta del entorno de las organizaciones y de su desplazamiento en el tiempo. El hombre actúa como centro de los procesos y ello hace poco predecible los mismos. Los patrones históricos de comportamiento no son la mejor guía sobre lo que cabe esperar en el futuro, ya que son las relaciones las que marcan las tendencias del sistema, y éstas son complejas y están altamente relacionadas entre sí. De ahí que la utilización de técnicas estadísticas tradicionales no pueda ser considerada lo suficientemente útil para explicar fenómenos en que las interacciones son la base de sistemas complejos que con el tiempo sufren alteraciones en nivel y ritmo diferentes. La búsqueda de este objetivo nos conduce a la necesidad de buscar explicaciones formales desde enfoques alternativos. 


\section{PROPOSICIONES}

\subsection{LOS RESULTADOS DE LA GESTIÓN MEDIOAMBIENTAL}

Se considera que la responsabilidad medioambiental de las corporaciones son un conjunto de iniciativas de la organización que tienen como objetivo, entre otros, mitigar el impacto de las empresas sobre el medio ambiente (Bansal y Roth, 2000; Hart, 1997). Ante este planteamiento, las respuestas estratégicas de las organizaciones puede concretarse en tres: (1) cumplimiento, (2) ir más allá del cumplimiento, y (3) la excelencia. Categorización de las motivaciones que conforma también los objetivos últimos de las respuestas estratégicas (Winn y Roome, 1993).

El "cumplimiento" implica el mero acatamiento de los requerimientos legales y la normativa existente. El "ir más allá del cumplimiento" hace referencia a aplicar políticas medioambientales no exigidas directamente por la legislación. Y la "excelencia", la búsqueda de la mejora, innovación y la consideración global de la gestión buscando la superación a la vez que se tienen en cuenta los intereses de todas las fuerzas interesadas en la actividad empresarial (es decir, teniendo en cuenta a los grupos de interés), admitiendo la responsabilidad social como un resultado clave de la gestión de la organización.

En consecuencia, existen tres respuestas estratégicas que implican también posturas que se desarrollan en un orden secuencial. El primer acercamiento es la preocupación por el cumplimiento de la normativa, su asunción y su sistematización en la gestión. Al igual que se puede hacer con los temas fiscales en donde se cumple la norma pero se busca que dicho cumplimiento sea lo menos costoso posible y altere al mínimo la actividad básica de la organización. Cuando se adquiere una conciencia de la importancia del ambiente y la necesidad de respetarlo, las organizaciones empiezan dando pasos para conseguir resultados más allá de lo que establece la normativa vigente. Cabe esperar que, a largo plazo, la gestión medioambiental desarrolle sus estrategias vinculadas a la excelencia en los resultados. Estos resultados incluirán la mejora del conocimiento para la toma de decisiones de la organización, la mejora de la formación de los empleados en cuestiones medioambientales, la mejora de la moral de los empleados, la mejora de la reputación de la organización, la mejora de las relaciones con la administración pública y comunidad local, la mejora de las relaciones con los medios de comunicación y la mejora de las relaciones con accionistas y trabajadores. Todas estas cuestiones se encuentran ligadas a una gestión de calidad que acercarán a la organización a la excelencia.

La gestión medioambiental avanza en un proceso de maduración que implica el paso por las fases previas. Una vez que una organización no sólo es excelente sino que busca la excelencia como una cuestión básica en su gestión, se puede hablar de maduración lo que implica el desarrollo de una gestión avanzada y compleja que tiene en cuenta todas las cuestiones relevantes, todos los intereses y sus relaciones a la vez proporcionando resultados adecuados para todos ellos. Evidentemente, esto sólo se construye con el tiempo. 
Proposición 1.Una gestión medioambiental madura se vincula a la obtención de resultados conectados con la mejora y la calidad en la gestión.

\subsection{EL EJE DE LA GESTIÓN MEDIOAMBIENTAL}

La gestión medioambiental se apoya en un número significativo de variables que se orientan a corto plazo para conseguir unas condiciones de producción y coste que no haga inviable la gestión de la organización y la venta de sus productos. El impacto de estas condiciones de producción y coste es la primera e inicial preocupación de una política de gestión medioambiental cuando ésta surge. Las condiciones básicas que sirven inicialmente de referente incluyen la reducción de los costes de aprovisionamiento (materias primas o componentes), de producción y los asociados al incumplimiento de la normativa medioambiental, así como la mejora de la productividad, la innovación en procesos y productos y la calidad de los productos.

Proposición 2. Las variables clave que condicionan la gestión medioambiental se encuentran ligadas a la producción y el coste de la misma.

\subsection{LA EVOLUCIÓN DE LA GESTIÓN MEDIOAMBIENTALY SU ESTABILIDAD}

El sistema de gestión medioambiental (EMS) es el proceso de identificar un conjunto de estrategias ambientales y de los procesos de la empresa establecimiento un buen alineamiento de ambos. Siendo la planificación un instrumento apropiado para desarrollar adecuadamente un sistema de gestión medioambiental ya que el sistema implica un considerable aumento de la complejidad de la gestión y la necesaria coordinación de los procesos y políticas.

En general, las organizaciones que han alineado sus procesos de gestión ambiental con los básicos del negocio, consideran varios prerrequisitos básicos para su realización: (i) Definir una estrategia básica para el diseño y desarrollo del EMS. (ii) Implementar el EMS mediante cambios en la organización de los recursos, restricciones y condicionamiento de los mismos. (iii) Evaluar el impacto del EMS sobre la ventaja competitiva de la empresa.

En la definición del sistema de gestión medioambiental (EMS), se han considerado los siguientes constructos:

- TEM (Total Enviromental Management). Recoge en qué medida la organización ha modificado sus prácticas de negocio con el objeto de reducir su impacto medioambiental, y en qué grado está comprometida en el desarrollo de acciones orientadas a la restauración del medioambiente.

- Organización Medioambiental. La gestión medioambiental es una elección estratégica y una oportunidad para la mejora de la organización, por lo que los objetivos, estrategias, políticas y planes medioambientales deben estar integrados en los generales de la organización y dirección y, en consonancia, mostrará un apoyo y una implicación decidida respecto a las cuestiones medioambientales, convirtiendo la gestión medioambiental en una cuestión de máxima. La organización adaptará su estructura interna y procedimientos orientados a 
prevenir y responder a los problemas medioambientales, invirtiendo en formación e implicación de los empleados sobre estas cuestiones.

- Compromiso de actuación medioambiental. El compromiso va más allá de las actuaciones o las políticas, representa una opción personal no sólo de la dirección sino también del conjunto de los empleados de la organización.

- Plazo de cambio. Los cambios no siempre son posibles en el corto plazo. La repercusión del tiempo es esencial para entender la gestión y sus resultados. Los cambios en la estrategia y las actuaciones a veces son radicales, rápidos y modifican los aspectos básicos de la organización. Sin embargo, los cambios se deben a modificaciones sustanciales en las estructuras formales de autoridad o a modificaciones sustanciales en los principios, valores y creencias de la organización, que evidentemente sólo a largo plazo pueden alcanzarse.

- Ritmo de cambio. Los cambios en la organización se deben, muchas veces, a improvisaciones diarias y pequeñas mejoras en los procesos de trabajo o relaciones sociales que se acumulan en el tiempo.

La eficacia y la eficiencia de los parámetros planteados y procesos del EMS están fuertemente ligados a un ambiente estable y a la calidad de la planificación de los recursos (Pun et al, 1998). Sin embargo, la gestión medioambiental madura a través de un proceso progresivo. Mientras no se alcance esa madurez las relaciones entre variables y la estructura del modelo correspondiente representan posiciones sin estabilizar.

Proposición 3. La estabilidad de un modelo de gestión medioambiental sólo se alcanza al final de un proceso de evolución y desarrollo en el que el tiempo y la acumulación de herramientas de gestión son básicos.

\subsection{LA POSTURA DE LA DIRECCIÓN Y SU RESPUESTA ESTRATÉGICA}

La postura de la dirección es la actitud que la dirección adopta respecto de las cuestiones estratégicas. Se concreta en la forma en que actúan los directivos frente a su entorno creando sus estrategias. Las posturas de la dirección son respuestas estratégicas diferentes y, en consecuencia, la obtención de resultados distintos ligados a la efectividad de dichas estrategias y a los objetivos que las enfocan.

Las empresas adoptan diferentes posturas que pueden agruparse en cuatro categorías (Brockhoff et al., 1999):

- Defensiva. Consiste en dar una respuesta positiva a las normas y regulaciones existentes, y mantienen la capacidad de reacción ante las nuevas regulaciones y estándares. Las investigaciones realizadas (Pun, 2002) señalan que las empresas que se suelen ubicar en este categoría son las más grandes, siendo muy pocas las que tienen una política ambiental explícita. Su comportamiento se explica en parte por las elevadas inversiones que realizan en tecnología y productos.

- Escapista. La adoptan empresas que consideran que deben salir de sus actuales negocios y explotar nuevos mercados involuntariamente. Le dan gran importancia al cumplimiento de la regulación pero no se preocupan por anticipar los nuevos estándares y no están pendientes de su evolución para antici- 
parlos. Acostumbran a tener una reducida dimensión y casi ninguna desarrolla una política ambiental explícita.

- Inactiva. Se trata de empresas cuya actividad implica pocos riesgos relacionados con el medio ambiente y se encuentran muy poco afectadas por regulaciones o presiones externas respecto a estos temas. Acostumbran a ser empresas de dimensión media.

- Activista. Sus actitudes son las contrarias a la postura inactiva, identificando más empresas en esta postura. La mayoría ha desarrollado una política de gestión ambiental. Cumplen la normativa, se interesan por los nuevos estándares y regulaciones y le dan una alta importancia a explotar nuevos mercados. Son proactivos con el objetivo de ir y escapar de muchas de las restricciones existentes. Introducen las cuestiones ambientales en la formulación de su estrategia y cambian la planificación de sus productos, sus compras, la fabricación y la logística de acuerdo con este enfoque (Azzone et al., 1997). La literatura sugiere que una parte importante del éxito de los nuevos productos está ligado a la integración de la estrategia de fabricación con la gestión medioambiental (Angell y Klasssen, 1999).

Proposición 4. La postura de la dirección respecto al cambio es el principal factor de influencia sobre los resultados buscados y las estrategias desarrolladas para alcanzarlos.

\subsection{LA INFLLUENCIA DE LOS GRUPOS DE INTERÉS (STAKEHOLDERS) EN LA GESTIÓN AMBIENTAL}

En las últimas décadas la comunidad nacional e internacional ha tomado conciencia de la importancia de cuidar el medioambiente. Los movimientos ecológicos cada más organizados se han dotado de capacidad analítica y de denuncia de las agresiones al entorno natural. Su fuerza ha ido creciendo tanto en el campo de la credibilidad ante la comunidad como por la atención de los medios de comunicación.

Los grupos interesados en la organización (stakeholders) tales como los clientes, comunidades locales, grupos ecologistas o gobiernos, presionan a las empresas para que consideren el impacto de sus acciones sobre el medioambiente (Berry y Rondinolli, 1998). La propia estrategia de la organización, pendiente de su entorno hace que muchos directivos den una respuesta responsable a los temas ambientales al advertir el impacto negativo de la opinión pública. A pesar de ello, no se encuentran posiciones de presión evidentes por parte de los accionistas de las empresas afectadas (Lawrence y Morell, 1995).

De todos los grupos interesados, el análisis demuestra que la importancia de la legislación es el factor de mayor influencia para que las empresas adopten un comportamiento ecológico responsable, tratando de evitar las penalizaciones y los costes asociados a la exigencia por medios legales del cumplimiento de la norma (Lampe et al., 1991; Lawrence y Morell, 1995; Vredenburg y Westley, 1993)

En esta investigación se considera que la influencia de los stakeholders se produce a través de tres tipos de agrupaciones: Grupos normativos (gobierno, asociaciones profesionales, grupos políticos), grupos organizacionales (consumidores, prove- 
edores, empleados y accionistas o propietarios) y grupos sociales (asociaciones de la comunidad, entidades medioambientales y medios de comunicación).

Proposición 5. La influencia de los stakeholders respecto a las cuestiones medioambientales es siempre un factor relevante pero cuando la organización desarrolla planes en el campo medioambiental su papel se vuelve determinante para definir y alcanzar los resultados de la gestión en esta materia.

\subsection{LOS OBJETIVOS PARA LA REDUCCIÓN DEL IMPACTO DE LA ORGANI- ZACIÓN SOBRE EL MEDIOAMBIENTE}

Generalmente, los objetivos ligados a la reducción del impacto de las organizaciones sobre el medioambiente han incluido: el control de la polución, la minimización de los desechos, la reducción del consumo de energía y la prevención de la polución (Bansal y Roth, 2000). Sin embargo, esto no es suficiente y los siguientes pasos consisten en la introducción de tecnologías limpias y el empleo de recursos ecológicamente sostenibles (Hart, 1997).

A efectos de esta investigación, se entiende que la preocupación inmediata en las políticas de gestión medioambiental son aquellas que tienden a reducir el impacto de la organización en tres cuestiones fundamentales: reducción de desperdicios y emisiones, reducción de materiales no renovables y reducción del uso energético. Una vez la la organización lleva tiempo desarrollando políticas sobre las cuestiones anteriores, los objetivos y las políticas se convierten en un hábito, de tal modo que se aceptan como algo consustancial con dicha organización. En este momento, estas políticas pasan a ser un instrumento para llegar a alcanzar otros objetivos de mayor (como por ejemplo, la excelencia en la gestión). De este modo, las empresas que pueden proporcionar simultáneamente calidad junto a productos ambientalmente amigables, tienden a disponer de un mayor potencial de conseguir mayores porcentajes de participación en el mercado (Pun et al., 1998).

Proposición 6. Los objetivos de actuación directa en los procesos de gestión medioambiental pierden con el tiempo su carácter finalista para convertirse en variables instrumentales pasando a ocupar su lugar los objetivos ligados a la excelencia de gestión (calidad, ambiente y responsabilidad social).

\section{METODOLOGÍA DE LA INVESTIGACIÓN}

Dado que el objeto de estudio obliga a estudiar la complejidad y llegar a conclusiones de futuro, se establece que el análisis de prospectiva es una herramienta adecuada para dicho objeto de análisis (Morin, 1998). Esta metodología ofrece la posibilidad de describir un sistema con ayuda de una matriz que relaciona todos sus elementos constitutivos (Bas, 1999). La herramienta informática que se utiliza para este análisis es MICMAC, la cual permite identificar las principales variables influyentes y dependientes, y establecer las variables esenciales para la evolución futura del sistema objeto de estudio (Godet, 1991). 
El análisis de prospectiva estructural se ha realizado por un grupo de trabajo compuesto por expertos con experiencia demostrada. Se buscó la participación de tres expertos en políticas medioambientales y especializadas en distintos campos de conocimiento. En los tres casos se trató de profesores doctores de diferentes universidades. El papel de estos expertos no consistió en identificar las variables (les fueron facilitadas por el investigador en base a la literatura existente) sino que debieron establecer las relaciones de dependencia e influencia entre las variables facilitadas.

A través del análisis estructural se relacionaron las variables en un tablero de doble entrada o matriz de relaciones directas. El grupo de expertos calificó los cuadrantes de la denominada matriz del análisis estructural mediante una escala numérica ligada a conceptos cualitativos. Para ello, para cada pareja de variables se plantearon las cuestiones siguientes: ¿existe una relación de influencia directa entre la variable i y la variable j? Para sus respuesta se les facilitó la utilización de una escala simple, si dicha respuesta es que no, se anota 0 , en el caso contrario, se plantea la opción de si esta relación de influencia directa es, débil (1), mediana (2), fuerte (3) o potencial (P). A partir de esta fase de calificación de la matriz se plantea a propósito de $\mathrm{n}$ variables, $\mathrm{n}$ por $\mathrm{n}-1$ preguntas, algunas de las cuales hubieran caído en el olvido a falta de una reflexión tan sistemática y exhaustiva. Este procedimiento de interrogación hace posible no sólo evitar errores, sino también ordenar y clasificar ideas.

Los expertos trabajaron individualmente para evitar que el contacto personal ejerciera una influencia no deseada basada en un sistema de relaciones. Posteriormente remitían al investigador sus evaluaciones sobre las relaciones de las variables. Éste comprobaba las coincidencias, que eran consideradas como definitivas, y las diferencias que eran de nuevo remitidas a los expertos para una nueva consideración, en función de la opinión de los demás expertos. Tras aplicar este procedimiento repetidas veces, en la cuarta iteración del procedimiento se llegó a una matriz consensuada.

\section{RESULTADOS DE LA INVESTIGACIÓN}

\subsection{EL ESCENARIO INICIAL. MATRIZ DE INFLUENCIA DIRECTA (MID)}

El resultado directo del trabajo de los expertos es una matriz (véase Tabla 1), en la cual cada línea representa la influencia de una variable sobre las demás. El total de las cuadrículas en línea indica la importancia de la influencia de una variable sobre el conjunto del sistema (nivel de influencia o motricidad directa). El total en columna indica el grado de dependencia de una variable (nivel de dependencia directa). De la matriz se desprende el modelo de influencias directas (figura 1). 
Tabla 1: Matriz de influencia-dependencia (MID)

\begin{tabular}{|c|c|c|c|c|c|c|c|c|c|c|c|c|}
\hline & 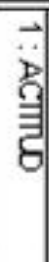 & 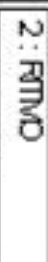 & 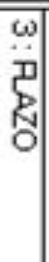 & 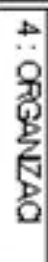 & 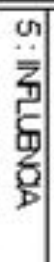 & $\begin{array}{l}\text { क } \\
\text { 田 } \\
\end{array}$ & 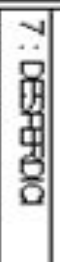 & 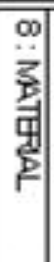 & 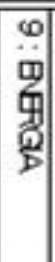 & $\begin{array}{l}\overrightarrow{0} \\
8 \\
8 \\
7 \\
7 \\
\frac{1}{8} \\
\overline{8}\end{array}$ & $\begin{array}{l}\overrightarrow{\vec{x}} \\
\overrightarrow{8} \\
\overrightarrow{\mathrm{b}}\end{array}$ & $\begin{array}{l}\vec{N} \\
\frac{8}{8} \\
B\end{array}$ \\
\hline 1: Actitud hacia el cambio & 0 & 3 & 3 & 2 & 0 & 3 & $\mathrm{P}$ & $\mathrm{P}$ & $\mathrm{P}$ & $\mathrm{P}$ & $\mathrm{P}$ & $\mathrm{P}$ \\
\hline 2: Futrmo de cambio & 0 & 0 & 2 & 1 & 0 & 0 & 1 & 1 & 1 & 0 & $\mathrm{P}$ & $\mathrm{P}$ \\
\hline 3: Pazo del cambio & 0 & 2 & 0 & $\mathrm{P}$ & D & 2 & 2 & 2 & 2 & 3 & 3 & 3 \\
\hline 4: Organización medio arrbiental & 0 & 2 & 3 & 0 & 1 & 2 & 3 & 1 & 2 & 1 & $\mathrm{P}$ & $\mathrm{P}$ \\
\hline 5: Influencia grupos & 2 & 2 & 2 & 2 & 0 & 2 & 2 & 2 & 2 & 2 & $\mathrm{P}$ & $\mathrm{P}$ \\
\hline 6: TEM & 0 & 1 & 1 & 2 & 1 & 0 & 2 & 2 & 2 & 2 & $\mathrm{P}$ & $\mathrm{P}$ \\
\hline 7 : Reducción desperdicios y emisiones & 0 & 1 & 1 & 1 & 0 & 0 & 0 & 2 & 2 & 1 & 3 & 3 \\
\hline 8: Reducción material no renov able & 0 & 1 & 1 & 1 & 0 & 0 & 2 & 0 & 2 & 1 & 3 & 3 \\
\hline 9: Reducción uso energético & 0 & 1 & 1 & 1 & 0 & 0 & 2 & 2 & 0 & 1 & 3 & 3 \\
\hline 10: Compromiso actuación ambiental & 0 & 2 & 2 & 2 & 0 & 3 & 3 & 3 & 3 & 0 & 2 & 2 \\
\hline 11: Resultados en costes & 2 & 2 & 2 & 2 & $\mathrm{P}$ & 2 & 3 & 3 & 3 & 3 & 0 & 2 \\
\hline 12: Resultados en calidad & 0 & 0 & 1 & 1 & 2 & 1 & 0 & 0 & 0 & 1 & 1 & 0 \\
\hline
\end{tabular}

El modelo, aunque en su expresión gráfica sólo presenta las relaciones más intensas, muestra unas relaciones complejas con una visión simplista de la política de medioambiente basada en cuestiones elementales y directas (véase figura 1).

Figura 1: Grafico de influencias directas del modelo gestión medioambiental

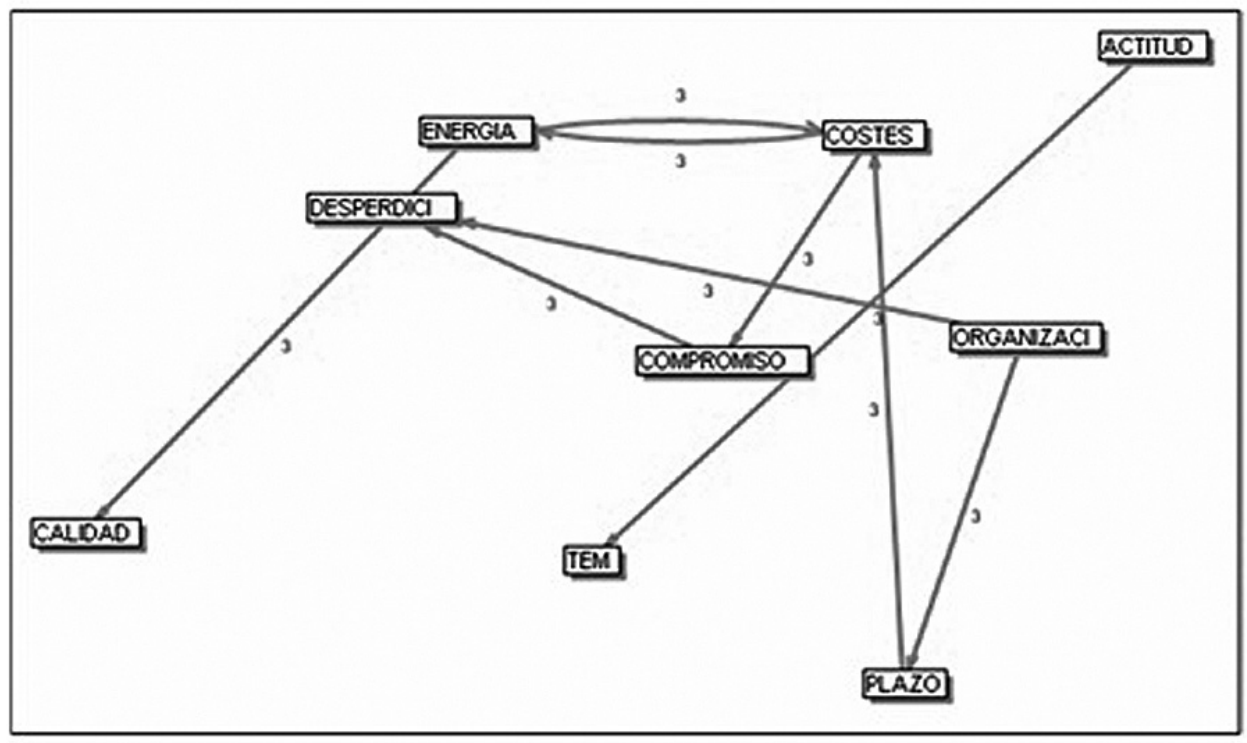


En primer lugar, se identifica una influencia directa entre la postura de la dirección y el grado en que: (i) modifica sus prácticas de negocio con el objetivo de reducir el impacto en el hábitat natural; (ii) desarrolla acciones orientadas a la restauración del medioambiente (TEM). Se trata por tanto, de que inicialmente la formación, sensibilidad y creencias de la dirección, es la responsable de la postura que realmente adopte la organización ante el medioambiente. Por encima de otras cuestiones ligadas a la racionalidad, los intereses económicos o los objetivos de la organización, la actitud o postura de la dirección es básica para desarrollar una gestión del ambiente como política empresarial.

En segundo lugar, el modelo indica que el modo en que está estructurada la organización es decisivo, tanto para los plazos de cambio en la gestión medioambiental como en la consecución de objetivos esenciales en relación con los desperdicios.

En tercer lugar, el modelo consiste en una cadena de relaciones que contribuyen decisivamente a la reducción de los desperdicios nocivos (uno de los objetivos básicos de la gestión medioambiental). De acuerdo con los resultados, los plazos para cambiar tienen una influencia decisiva sobre los costes y el compromiso de la dirección está condicionado por los costes. Los directivos son capaces de aumentar su compromiso si los costes son bajos pero pierden dicho compromiso si son costes son altos. Por tanto, el grado de compromiso afecta a los objetivos relacionados con la reducción de emisiones y desperdicios.

En cuarto lugar, se desprende que los factores iniciales del proceso de gestión medioambiental, se encuentran en dos variables, una ligada a los líderes de la empresa y, la otra, la propia capacidad de organizarse de la empresa. La capacidad de organización se desarrolla a lo largo del tiempo, una buena estructura organizativa con sistemas y procedimientos de gestión asentados no surge espontáneamente sino que es el resultado de un proceso de crecimiento. Por ello, las políticas de gestión medioambiental requieren que las empresas dispongan de organizaciones modernas y con un alto nivel de desarrollo. La consecuencia para la política es que las organizaciones simples y mal estructuradas difícilmente serán capaces de desarrollar políticas de gestión medioambiental.

En quinto lugar, otra cuestión de interés es el bucle entre costes y reducción de la energía. La reducción del consumo energético se convierte en un objetivo que depende directamente de los costes de dicho consumo y no estrictamente en un objetivo de gestión medioambiental.

Finalmente, es significativo que la calidad surja como una cuestión final, como resultado de lo anterior y no como un objetivo directo de la gestión medioambiental.

\subsection{PLANO DE INFLUENCIAS Y DEPENDENCIAS}

Los resultados obtenidos se pueden representar en un plano las influencias y dependencias directas entre variables (Figura 2). Las coordenadas de las variables corresponden a las sumas de las influencias y las dependencias calculadas a partir de la matriz MID. Esta representación permite orientar aspectos de la estrategia organizacional e identificar de un modo visual dicha estrategia. 
En la zona superior derecha, se encuentran las variables-clave o variables-reto del sistema. Variables muy motrices y muy dependientes que perturban el funcionamiento normal del sistema y sobre determinan el propio sistema. Por naturaleza son inestables y se corresponden con los retos del sistema, lo que las convierte en variables de extraordinaria importancia e integrantes del eje estratégico. Estas variablesclave son: los costes, el plazo y el compromiso de la dirección.

Figura 2: Plano de influencias y dependencias del modelo gestión medioambiental

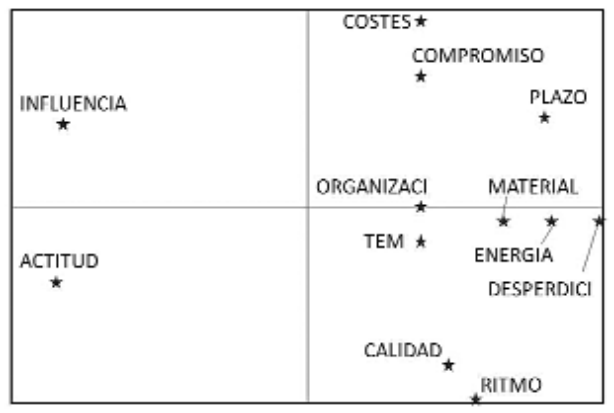

En la zona superior izquierda, se encuentran la influencia como variable determinante, poco dependiente y muy motriz, según la evolución que sufra a lo largo del periodo de estudio se convierte en freno o motor del sistema, de ahí su denominación. El papel de la influencia de los grupos interesados en el medioambiente queda identificado como un elemento motriz importante y por tanto, su evolución requiere una atención especial.

En la figura se identifica una serie de variables que se posicionan en un lugar sin una definición clara para la estrategia, estas son: organización, material, desperdicios, energía y TEM. Ello pone de manifiesto la falta de claridad de los gestores y expertos sobre de cuáles son y deben ser las orientaciones concretas de la gestión medioambiental. Es decir, refleja la posición ambigua de muchas organizaciones respecto al medioambiente. El debate entre intereses, presión institucional y conciencia social, posiblemente no esté resuelto, lo demuestra que las estrategias no se apoyan de un modo consciente y regular sobre variables a las que se les asigna un papel como palanca o regulación en el modelo.

Las variables de resultados están claramente identificadas en los resultados de calidad y el ritmo con que se producirá el cambio. Esta última, resulta difícil de considerar como un objetivo, su posición es, sin duda, una anomalía que sólo puede explicarse por la semi-desarticulación de las políticas medioambientales en los procesos que no llevan emprendidos mucho tiempo, procesos que intentan evitar únicamente los problemas institucionales derivados de la falta de cumplimiento de la normativa existente. 


\subsection{LA EVOLUCIÓN DE LA ESTRUCTURA DEL MODELO}

Para realizar un análisis prospectivo se han de tener en cuenta las relaciones potenciales, es decir, aquellas que son inexistentes hoy pero que la evolución del sistema hace probables o por lo menos posibles en un futuro más o menos lejano. Además, es preciso descubrir las relaciones indirectas que generan con el tiempo un fuerte efecto sobre las relaciones y la estructura del modelo.

Descubrimos las variables ocultas, gracias a un programa de multiplicación matricial (MICMAC) aplicado a una clasificación directa.

Toda matriz debe converger hacia una estabilidad al cabo de un cierto número de interacciones, generalmente de cuatro o cinco para una matriz de tamaño 50. En ausencia de criterios establecidos matemáticamente, se tiene en cuenta el número de permutaciones necesarias a cada interacción para clasificar el conjunto de variables en influencia y en dependencia. En consecuencia, Micmac selecciona las variables con el fin de obtener la clasificación directa.

\subsection{GRAFICO DE INFLUENCIAS INDIRECTAS POTENCIALES}

El gráfico de influencias, dentro de la visión prospectiva, permite visualizar las relaciones más importantes entre variables. En dicho gráfico se observan dos cuestiones: (i) La mayor complejidad de las relaciones, superior a las derivadas del gráfico de influencias directas. (ii) La coherencia superior de los resultados de las relaciones con los enfoques teóricos básicos. Esta última cuestión se encuentra en paralelo con las apreciaciones realizadas en las proposiciones anteriores, pues la coherencia entre fines y medios la proporciona la estrategia y el sistema de gestión ambiental que no existen hasta que la organización alcanza, con el tiempo, un grado suficiente de madurez en el tratamiento de estos temas.

Figura 3: Gráfico de influencias indirectas potenciales del modelo gestión medioambiental

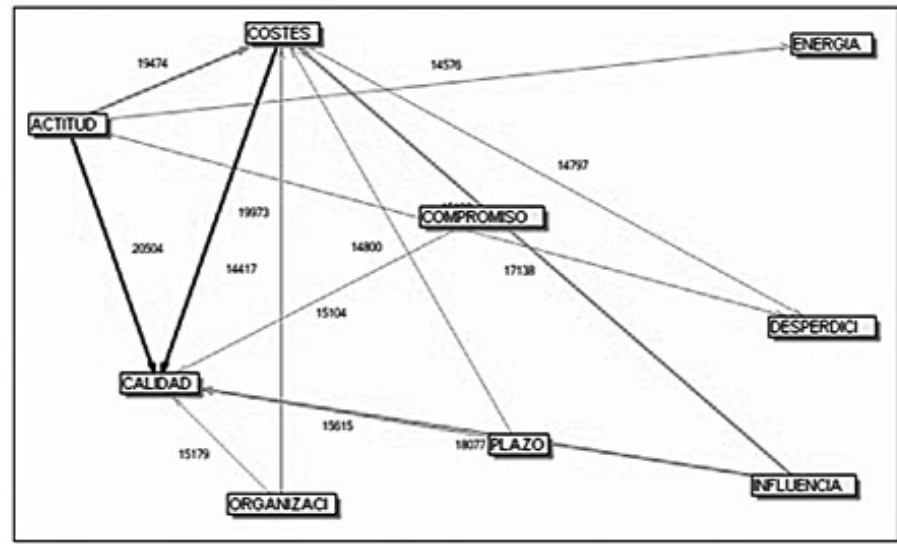

$$
\begin{aligned}
& \text { Ifluencias mós debles } \\
& \text { - ifluenciss debles } \\
& \text { - influencias medias } \\
& \text { - infuenciss relotivanerte inportarte: } \\
& \text { - influenciss mós inoortartes }
\end{aligned}
$$


4.5. Pl a no de in Fl uencias/dePendencias indirectas Pot enciales

1 as estrategias se vinculan a las acciones realizadas sobre unas variables con capacidad de influencia. $\mathrm{n}$ inguna variable es irrelevante pero no todas tienen la misma capacidad para generar progreso mediante cambios en los procesos. el valor estratégico de cada variable está determinado por la suma de su valor de su motricidad o influencia y de su valor de dependencia respecto a los movimientos de otras variables (Godet, 1979). Para cualquier variable su valor estratégico estaría determinado por la suma de su valor de motricidad (influencia) y de su valor de dependencia.

la primera diagonal (superior izquierda a inferior derecha) es la diagonal de estratégica (se la describe en muchos estudios de prospectiva como de entradas y salidas) y aporta el sentido de lectura del sistema. la segunda diagonal es la diagonal de gestión, ya que cuanto más se aleja del origen, mayores son los condicionamientos para la gestión que tienen las variables. la segunda diagonal reparte el plano entre las variables motrices o influyentes (por encima de la diagonal) y las dependientes (debajo de la diagonal).

Figura 4: Plano de influencias y dependencias indirectas potenciales del modelo gestión medioambiental

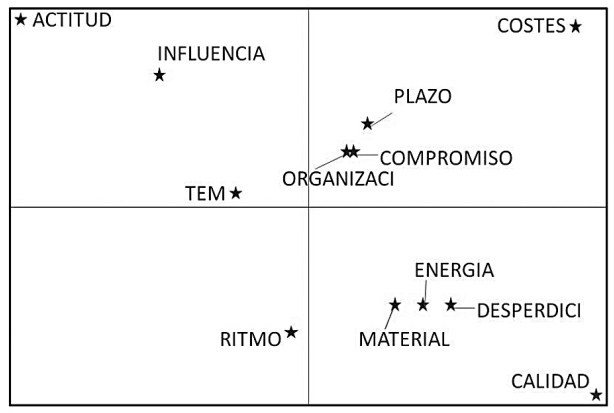

en la parte superior izquierda se sitúan las variables de entrada o determinantes, fuertemente motrices, poco dependientes, y que determinan el funcionamiento del sistema. la actitud de la dirección se convierte en el elemento motor del sistema que además tiene como apoyo la influencia de los stakeholders.

en el centro se sitúan las variables reguladoras que participan en el funcionamiento normal del sistema en este caso teM.

abajo y a la derecha figuran las variables de salida. dan cuenta de los resultados de funcionamiento del sistema y son poco influyentes y muy dependientes. se les califica igualmente como variables resultado o variables sensibles. se pueden asociar a indicadores de evolución, pues se traducen frecuentemente como objetivos. los objetivos de reducción tanto de materiales (desperdicios y energía) se encuentran agrupados en un nivel intermedio (palancas de gestión), mientras que la calidad se convierte en un objetivo finalista, situado en el extremo del gráfico. 
El reparto de las variables en la segunda diagonal permite establecer la siguiente clasificación por tipologías de variables:

- Zona próxima al origen: variables autónomas. Son poco influyentes y poco dependientes, y se corresponden bien con tendencias pasadas o inercias del sistema bien están desconectadas de él. Por tanto, no constituyen parte determinante para el futuro del sistema. Es preciso remarcar que no es que carezcan de importancia sino que, comparativamente, los esfuerzos que se destinen ofrecerán mejores resultados en variables situadas en los otros grupos. El mapa no sitúa ninguna variable en esta zona.

- Zona superior de la diagonal: variables-clave. Son muy motrices y muy dependientes. Perturban el funcionamiento normal del sistema, y sobre-determinan el propio sistema. Son por naturaleza inestables y se corresponden con los condicionantes esenciales del sistema, lo que las convierte en variables de extraordinaria importancia e integrantes del eje estratégico. De una forma muy clara aparecen los costes como la variable clave del sistema.

- Zona central del plano: variables reguladoras. Se convierten en "llave de paso" para alcanzar el cumplimiento de las variables-clave y que éstas vayan evolucionando tal y como conviene para la consecución de los objetivos del sistema. Determinan el funcionamiento del sistema en condiciones normales. En este caso, son variables reguladores: TEM, organización, compromiso y plazo.

A continuación procedemos a comentar las proposiciones propuestas en el estudio. La Proposición 1, indica que una gestión medioambiental madura se vincula a la obtención de resultados conectados con la mejora y calidad en la gestión. En este punto, la experiencia y la evolución de las principales organizaciones españolas son un gran indicador al respecto. Ejemplo de la evolución de las organizaciones en esta línea y no por planteamientos de índole teórica, es el cambio de denominación del Club de Excelencia en Gestión que pasó de denominarse Club Gestión de Calidad debido a la relevancia que adquirieron los aspectos de responsabilidad social y gestión medioambiental. Esta realidad se ve corroborada por el análisis de prospectiva que se ha realizado, la evolución de la calidad como centro de los objetivos de la gestión medioambiental. El rol de los objetivos operativos como palanca para conseguir resultados de calidad, es una prueba de ello que surge en un proceso de evolución.

Los gestores siempre tienen en cuenta cuestiones de producción y de costes, siendo la gestión medioambiental frecuentemente calificada como coste. Un coste que habría que asumir por condicionamientos legales o de responsabilidad social pero que, a su vez, se debería minimizar para que no afectara de forma significativa a los resultados, poniendo en peligro la estabilidad económica de la organización. Progresivamente, los gestores han ido asumiendo la importancia de la responsabilidad social y han incluido la gestión medioambiental en su agenda. A este respecto y en relación a los resultados del análisis estructural, tanto en el momento actual como el resultado futuro, confirman la Proposición 2, señalando que la variable clave que condiciona la gestión medioambiental se encuentra ligada a los costes del sistema productivo. 
La madurez de las organizaciones se alcanza siempre después de un proceso en el que el tiempo es indeterminado. La cuestión es contestar a la pregunta ¿Cuándo puede decirse que una organización ha alcanzado un elevado grado de madurez? La respuesta se encuentra en que la gestión camine dentro de un proceso de excelencia $\mathrm{y}$, al menos, haya llegado a un nivel que el modelo EFQM denomina compromiso con la excelencia.

Para que una empresa sea denominada excelente, y siguiendo los criterios EFQM debe reunir los siguientes requisitos: (i) La mejora continua es una de sus características y el aprendizaje y la innovación empiezan a formar parte de su modo de actuación. (ii) Los recursos humanos son valorados en toda su dimensión y se realiza una gestión de personal con políticas que contribuyen a su satisfacción y motivación. (iii) El director y su equipo actúan como auténticos líderes. (iv) Se dedican grandes esfuerzos a la gestión de los procesos. (v) El personal recibe la formación adecuada para el desempeño de su actividad. (vi) Se mide el nivel de satisfacción de los clientes y se tienen en cuenta las aspiraciones y satisfacción de los grupos interesados.

En términos generales, se podría señalar que las organizaciones con gestión medioambiental son las que desarrollan sus procesos clave, trabajan con normas ISO 9000 o anteriores, sus clientes les exigen la certificación y, por lo tanto, se desenvuelven en un entorno de responsabilidad social y un nivel de gestión evolucionado. Generalmente, se trata de medianas empresas en relación con el volumen de facturación (no con el número de empleados).

Todo lo anterior conduce a la confirmación de la Proposición 3, indicando que la estabilidad de un modelo de gestión medioambiental sólo se alcanza al final de un proceso de evolución y desarrollo en el que el tiempo y la acumulación de herramientas de gestión son básicos.

El papel del liderazgo ha sido señalado desde siempre como uno de los factores clave para entender sus estructuras, compromisos y forma en que realizan su gestión. Siguiendo con el modelo EFQM se puede decir que la gestión precisa el papel de líderes excelentes, entendidos como aquellos que desarrollan y facilitan la consecución de la misión y la visión, desarrollan los valores y sistemas necesarios para que la organización logre un éxito sostenido y hacen realidad todo ello mediante sus acciones y comportamientos. En periodos de cambio son coherentes con el propósito de la organización, y cuando resulta necesario, son capaces de reorientar la dirección logrando arrastrar tras ellos al resto de las personas. En consecuencia, según el EFQM los líderes definen e impulsan los cambios necesarios en la organización, en el modelo de la organización y en sus relaciones externas.

La importancia del liderazgo radica en que su influencia sobre la organización, su gestión y sus estrategias, es decisiva. Según cómo sea el liderazgo así serán sus estrategias y, por tanto, los resultados finales se verán afectados decisivamente. El modelo de prospectiva planteado no deja dudas acerca del papel de la postura de la dirección como factor determinante básico sobre los resultados de la gestión. No obstante, en el primer análisis en el que se refleja la realidad de la gestión en el momento actual, la postura de los directivos se muestra como una variable insignificante. 
Esta aparente contradicción tiene un sentido práctico de base real. Cuando se habla de líderes excelentes se está haciendo mención a unos líderes que crean y difunden valores sociales a la organización, que se desenvuelven en organizaciones en las que se distingue una identidad y una cultura propia. Son líderes para los que el compromiso social es un concepto asentado durante largo tiempo. Muy pocas organizaciones de las consideradas en la consulta realizada disponen de ese tipo de liderazgo.

La mayor parte de las organizaciones consideradas en la encuesta tienen una breve tradición en la introducción de medidas medioambientales y en la integración en sus estrategias con un papel relevante. Lo que indica el modelo de prospectiva es que partiendo de esa realidad en donde existen gestores medioambientales pero no líderes excelentes en este campo, cabe esperar una evolución de su actitud, de tal modo en que ésta llegue a convertirse en un factor decisivo para explicar la estrategia medioambiental de la organización. La confirmación de la Proposición 4, sobre la postura de la dirección respecto al cambio es el principal factor de influencia sobre los resultados buscados y las estrategias desarrolladas para alcanzarlos, se confirma como un factor de futuro en vez de como un reflejo de la situación actual.

El impacto de las organizaciones en la sociedad cada vez es más visible. La comunidad, en general, tiene necesidades y expectativas que quieren ver satisfechas a través de las organizaciones que operan en su entorno. De este modo, se generan grupos que se interesan por las organizaciones, ya que a través de ellas pueden mejorar su calidad de vida, el medio ambiente y la conservación de los recursos naturales. Esta consideración de grupos interesados engloba a los organismos e instituciones cuya opinión o actuación pueda afectar a la organización.

La existencia de grupos interesados obliga a las organizaciones a realizar medidas directas y periódicas, a través de encuestas o cualquier otro medio de consulta, de la percepción que tiene la comunidad de la organización y, a continuación, establecer objetivos respecto de aquellos aspectos que son relevantes (imagen corporativa, incidencia en la economía, relación con las autoridades, ética, grado de implicación en la educación, apoyo a actividades culturales o deportivas, actividades filantrópicas o de apoyo a ONG's, prevención de riesgos laborales, protección y preservación del medio ambiente o grado de reciclaje de sus productos, entre otros).

Diferenciando los tres tipos de grupos de interés se pueden extraer la siguiente información. Los grupos denominados normativos (gobierno o asociaciones profesionales) tienen capacidad para exigir que no exista ningún incumplimiento de las normas y leyes vigentes en la actividad empresarial. Los grupos organizacionales (clientes, proveedores, accionistas y empleados), en ocasiones, fuerzan a que se gestionen todas las cuestiones relativas al medio ambiente de acuerdo con alguna norma reconocida. Los grupos sociales (asociaciones de la comunidad o medios de comunicación) acostumbran a tener una influencia de calado (Díez et al, 2008). La persistencia de la presión por la vía de la vigilancia social y la protesta así como su aparición periódica en los medios, crea tensiones en las empresas que no pueden mantenerse a largo plazo, sin un fuerte deterioro de su imagen y de su capacidad de producción y servicio. 
Las empresas eligen diferentes estrategias en función de las presiones, que no varían sustancialmente entre países como Estados Unidos y Alemania (Brockhoff et al, 1999). Dentro de las presiones las de mayor importancia son: la relevancia $(5,3$ en una escala de 1 a 6 ), el interés de los consumidores $(4,3)$, el interés de los distribuidores $(3,2)$ y por último, las prácticas competitivas $(2,7)$.

La consecuencia es que las organizaciones acaban estableciendo objetivos en el campo medioambiental que integran dentro de sus planes y estrategias como un modo de responder a las presiones de los grupos interesados. Es en ese momento, cuando la organización desarrolla planes en el campo medioambiental, el papel de los grupos interesados se vuelve determinante para definir y alcanzar los resultados de la gestión en esta materia tal como se señala en la Proposición 5.

Inicialmente, las presiones reguladoras y de los consumidores centran la atención de las políticas medioambientales sobre temas como: reducción de desperdicios y emisiones, reducción materiales no renovables, y reducción uso energético. Revisando sus procesos de producción, las empresas reducen su impacto ambiental pero simultáneamente bajan sus costes de los inputs y eliminan parte de sus desechos (Cordano, 1993; Lampe et al., 1991; Porter y Van der Linde, 1995). Posteriormente, otros aspectos ligados a objetivos estratégicos generales comienzan a ser considerados por las organizaciones. Las pequeñas empresas consideran que el aumento en la participación en el mercado es uno de esos objetivos, sin embargo, la reducción de costes es todavía más importante y condiciona los objetivos de mayor calado. Los directivos con posturas más proactivas consideran que la calidad ecológica es un factor que influye fuertemente en la posición competitiva (Brockhoff et al, 1999). En función del tamaño de la empresa, por un lado, las pequeñas empresas, con recursos limitados, realizan sus cambios adoptando muchas veces estrategias de deslizamiento hacia nuevos mercados para evitar las presiones en materia medioambiental. Por otro, las grandes empresas pueden hacer frente a dichas presiones (Brockhoff et al, 1999).

El trabajo de Pun et al. (2002) demuestra que, entre las organizaciones que disponen de un sistema de gestión medioambiental, el principal beneficio que obtienen es la fuerte lealtad de los consumidores (más que el incremento de la imagen de la compañía). Se producen oportunidades y ventajas económicas y, como consecuencia, actuaciones corporativas responsables con su ambiente. Éstas se relacionan con objetivos como la reputación corporativa (Hart, 1995: Russo y Fouts, 1997), capacidades de aprendizaje (Bonifant et al, 1995; Hart, 1995), y calidad de los productos (Shrivastava, 1995).

De acuerdo con la Proposición 6, los objetivos de actuación directa en los procesos de gestión medioambiental pierden con el tiempo su carácter finalista para convertirse en variables instrumentales pasando a ocupar su lugar los objetivos ligados a la excelencia de gestión (calidad, ambiente y reputación, etc.).

\section{CONCLUSIÓN Y DISCUSIÓN}

Aunque son muchas las cuestiones que han sido discutidas en el trabajo, el análisis de prospectiva identifica una orientación evolutiva de las organizaciones sobre los aspectos medioambientales. Las organizaciones pasan por fases de asimilación y de 
interiorización de las cuestiones ecológicas que parten de un primer momento de rechazo y temor por la pérdida de eficiencia o por la reducción de sus beneficios. A continuación, si esa fase se supera la organización entiende la importancia de los temas ecológicos y busca legitimarse ante su entorno. En una tercera fase, la organización llega a convencerse que el interés ecológico también le proporciona ventajas competitivas que la pueden colocar en una mejor posición en el mercado. Por último, algunas organizaciones, muy pocas todavía, asumen su responsabilidad medioambiental como una ética de su negocio, subordinando cualquier consideración económica.

El presente trabajo no ha ido dirigido de forma esencial en esa dirección, es decir, no ha buscado la identificación de las fases de su comportamiento ambiental. Sin embargo, es una cuestión que ha surgido en el desarrollo del mismo, lo cual ayuda a explicar muchas cuestiones que de otro modo parecen difíciles de valorar

En un primer paso, las organizaciones se concentran en las cifras contables y la estimación para definir su respuesta ecológica, expresando su preocupación en función del gasto y en la creencia de que los consumidores no apreciarán el esfuerzo o la pérdida de cotización de la compañía. Estas firmas escogen las opciones que le aseguran los beneficios más altos, independientemente de sus consecuencias ecológicas.

Cuando la organización supera el paso anterior, se introduce en una segunda cuestión, su deseo de alcanzar la legitimidad. La legitimación de una firma se refiere al deseo de mejorar la idoneidad y corrección de sus acciones dentro de un juego establecido de regulaciones, normas, valores y creencias (Suchman, 1995). Obedecer la legislación, crear un comité o una dirección ambiental para supervisar los impactos ecológicos de una firma y aconsejar a la alta dirección, desarrollar redes o comités con representación de la comunidad local, realizar auditorías ambientales, establecer una sistema de respuesta en emergencias y alinear la firma con los defensores del medioambiente, son actuaciones que buscan la legitimación de la organización (Díez et al, 2010a). La carencia de legitimidad en una firma puede acabar con la licencia para operar o reducir su capacidad de supervivencia a largo plazo. Las organizaciones buscan su legitimidad en base a asegurar su sostenibilidad a largo plazo o su supervivencia e incluso, no perder su licencia de operar (Díez et al, 2010b). Estas observaciones respaldan la relación teórica entre la legitimidad y la supervivencia organizativa que sostiene la teoría institucional (Meyer y Rowan, 1977; Zucker, 1987). Los datos indican (Hart, 1997; Wood, 1991) que en la mayoría de los casos la legitimación ha ido dirigida a obedecer las normas y regulaciones institucionales. No se concentran en realizar esfuerzos previsores sino en reaccionar ante las restricciones externas para evitar las sanciones. Las organizaciones aspiran a cubrir los estándares más que a superarlos. Su objetivo es minimizar los riesgos y los costes, tratan de asegurar que sus respuestas ecológicas cubren las normas. El enfoque dominante de éstas es imitar a sus semejantes (Deniz y Garcia, 2011).

En una tercera fase se encuentra la posición en la que las valoran la gestión ambiental como instrumento para mejorar la competitividad, entendida como el potencial de la organización para dar una respuesta ecológica con objeto de mejorar la rentabilidad a largo plazo. De acuerdo con estudios previos (Bansal y Roth, 2000), las reacciones ecológicas que mejoran la competitividad incluyen la energía y la gestión de desperdicios. Las reducciones tienen como consecuencia en muchos casos el conseguir un nivel 
más alto de productos con los mismos recursos, comercialización basada en lo natural, un etiquetado como producto ecológico y desarrollo de ecoproductos.

Las organizaciones motivadas por la competitividad esperan que su capacidad de respuesta medioambiental influya en la consecución de una ventaja sostenida y mejore su rentabilidad a largo plazo. Habitualmente, las firmas compiten sobre precio y calidad, pero cada vez más compiten en temas ambientales, ya que es otro medio de obtener una ventaja competitiva. Muchas de las iniciativas emprendidas por estas empresas son simples operaciones de gestión que requieren cambios menores de los procesos pero que mejoran la eficiencia de dichas operaciones, lo que a su vez incrementa la competitividad debido a gastos más bajos como el apagar las luces por la noche o revisar el aire acondicionado cuidadosamente.

Con este objetivo emprenden actuaciones que buscan mejorar la reputación, la eficiencia de los procesos y la fiabilidad de los productos (Hart, 1995). Estas actuaciones se desarrollan a través de la comercialización basada en la ecología, menor utilización de los recursos, intensificación de los procesos y nuevo equipamiento de capital. Incluso, en muchos casos, es más fácil contratar a empleados de calidad ya que la organización obtiene una mejor reputación (Bansal y Roth, 2000). Bajo la motivación de la competitividad las iniciativas sociales son asumidas y se emprenden fundamentalmente cuando sirven para aumentar el rendimiento financiero a largo plazo de una firma. En cualquier caso, si la ciencia ambiental fuera más contundente a la hora de valorar y asegurar los impactos ecológicos de las actividades productivas y si los consumidores fueran más exigentes, las empresas muy probablemente mostrarían una capacidad de respuesta ecológica más grande.

Finalmente, las empresas motivadas por la responsabilidad ecológica, promueven un enfoque más holístico en relación con su respuesta ecológica. Las organizaciones valoran sus relaciones a largo plazo con el ambiente natural, no se centran en los intereses corporativos sino en promover el interés social (Lampe et al., 1991; Wood, 1991) porque consideran que esa es la manera correcta de actuar. La responsabilidad ecológica se basa en la motivación que proviene de la preocupación que tiene una organización por sus obligaciones sociales y valores. Las investigaciones destacan que las iniciativas motivadas por la responsabilidad ecológica incluyen la reconversión de terrenos en áreas verdes, la creación de una línea poco rentable de productos verdes, las donaciones para grupos de interés ecologistas y otras agrupaciones comunitarias locales, el uso de papel reciclado, la sustitución de artículos de consumo o productos de oficina por otros más benignos ecológicamente.

Una característica relevante de esta motivación es su preocupación para el bien social. Las empresas actúan con un sentido de obligación, de responsabilidad, o de filantropía con independencia de sus intereses particulares (Bucholz, 1991; L'Etang, 1995). Las empresas motivadas por la responsabilidad ecológica a menudo señalan a una persona dentro de la organización como aquella que claramente había liderado la respuesta ecológica de la misma. El proceso de decisión está a menudo basado en los valores de personas individuales o sobre los valores de la organización. Esta conclusión es compatible con los estudios de Lawrence y Morell (1995) y Winn (1995), que mostraban que la dirección superior de las empresas es la responsable del liderazgo de dirección ambiental. 


\section{REFERENCIAS BIBLIOGRÁFICAS}

ABRAHAMSON, E.; ROSENKOPF, L., 1993. Institutional and competitive bandwagons: Using mathematical modeling as a tool to explore innovation diffusion. Academy of Management Review, 18, 487-517.

ANGELL, L.C.; KLASSEN, R.D., 1999. Integrating environmental issues into the mainstream: an agenda for research in operations management. Journal of Operations and Production Management, 11 (3), 63-76.

BANSAL, P.; ROTH, K., 2000. Why companies go green: A model of ecological responsiveness. Academy of Management Journal, 43 (4), 717-736.

BAS, E., 1999. Prospectiva. Herramientas para la gestión estratégica del cambio, Ed. Ariel S.A. Barcelona.

BERRY, M. A.; RONDINOLLI, D. A., 1998. Proactive corporate environmental management: A new industrial revolution. Academy of Management Executive, $12(2), 1-13$.

BONIFANT, B. C.; ARNOLD, M. B.; LONG, F. J., 1995. Gaining competitive advantage through environmental investments. Business Horizons, 38 (4), 37-47.

BROCKHOFF, K.; CHAKRABARTI, A. K.; KIRCHGEORG, M., 1999. Corporate strategies in environmental management. Research Technology Management, 42 (4), 26-31.

BUCHHOLZ, R. A., 1991. Corporate responsibility and the good society: From economics to ecology: factors which influence corporate policy decisions. Business Horizons, 34 (4), 1-19.

CORDANO, M., 1993. Making the natural connection: Justifying investment in environmental innovation. Proceedings of the International Association for Business and Society, 530-537.

DENIZ, M; GARCIA, A., 2011. El isomorfismo mimético y las ventajas de localización como determinantes de la elección del modo de entrada. Revista Europea de Dirección y Economía de la Empresa, 20 (1), 105-122

DÍEZ, F.; MEDRANO, ML.; DÍEZ, E. 2008. Los grupos de interés y la presión medioambiental. Cuadernos de Gestión. Vol. 8. N.2, 81-96

DÍEZ, F.; BLANCO, A.; PRADO, C., 2010a. Legitimidad como factor clave del éxito organizativo. Investigaciones Europeas de Dirección y Economía de la Empresa, 16 (3), 127-143.

DÍEZ, F.; BLANCO, A.; PRADO, C., 2010b. Medición de la legitimidad organizativa: el caso de las sociedades de garantía recíproca. Cuadernos de Economía y Dirección de la Empresa. 43 (junio), 115-144

DIMAGGIO, P. J.; POWELL, W. W., 1983. The iron cage revisited: Institutional isomorphism and collective rationality in organizational fields. American Sociological Review, 48, 147-160.

DUPUY, 1982. Ordres et désordres. Seuil. Paris.

GLADWIN, T. N.; KENNELLY, J. J.; KRAUSE, T. S., 1995. Shifting paradigms for sustainable development: Implications for management theory and research. Academy of Management Review, 20, 874-907.

GODET, M., 1991. Prospectiva y planificación estratégica, S.G. Editores, Barcelona. 
GODET, M., 1979. Manuel de prospective estratégique, tome 2: L'art et la méthode, Dunod, Paris.

GODET, M., 2000. La caja de herramientas de la prospectiva estratégica. Cuadernos de LIPS, $\mathrm{n}^{\circ} 5$.

HART, S. L. 1995. A natural-resource-based view of the firm. Academy of Management Journal, 37, 986-1014.

HART, S. L., 1997. Beyond greening: Strategies for a sustainable world. Harvard Business Review, 75 (1), 66-76.

LAMPE, M.; ELLIS, S. R. ; DRUMMOND, C. K., 1991. What companies are doing to meet environmental protection responsibilities: Balancing legal, ethical, and profit concerns. Proceedings of the International Association for Business and Society, 527-537.

LAWRENCE, A. T.; MORELL, D., 1995. Leading-edge environmental management: Motivation, opportunity, resources, and processes. In D. Collins y M. Starik (Eds.), Research in corporate social performance and policy. GT: JAI Press Greenwich, 99-126.

L'ETANG, J., 1995. Ethical corporate social responsibility: A framework for managers. Journal of Business Ethics, 14, 125-132.

MEYER, J. W.; ROWAN, B., 1977. Institutionalized organizations: Formal structure as myth and ceremony. American Journal of Sociology, 83, 340-363.

MORIN, E., 1998. Introducción al pensamiento complejo, Gedisa editorial, Barcelona.

NEHRT, C., 1996. Timing and intensity effects of environmental investments. Strategic Management Journal, 17, 535-547.

PORTER, M. E.; VAN DER LINDE, C., 1995. Green and competitive: Ending the stalemate, Harvard Business Review, 73 (5), 120-134.

PRIGOGINE, I., 1990. Loi, histoire et desertion. La querelle du déterminisme, Collection le débat, Gallimard, Paris.

PUN, K.F.; FUNG, Y.K.; WONG, F.Y., 1998. Identification of critical factors for total quality environmental management, Proceedings of the 3rd Annual International Conference on Industrial Engineering Theories, Applications and Practice, Hong Kong, December, 114-119.

PUN, K.; HUI, I.; LAU, H.; LAW, H.; LEWIS, W. G., 2002. Development of an EMS planning framework for environmental management practices. The International Journal of Quality y Reliability Management, 19 (6/7), 688-700.

PURSER, R. E.; PARK, C.; MONTUORI, A., 1995. Limits to anthropocentrism: Toward an ecocentric organization paradigm? Academy of Management Review, 20, 1053-1089.

RUSSO, M. V.; FOUTS, P. A., 1997. A resource-based perspective on corporate environmental performance and profitability. Academy of Management Journal, 40, 534-559.

SHRIVASTAVA, P., 1995. The role of corporations in achieving ecological sustainability. Academy of Management Review, 20, 936-960. 
SHRIVASTAVA, P., 1994. Castrated environment: Greening organization studies. Organization Studies, 15, 701-720.

SUCHMAN, M. C., 1995. Managing legitimacy: Strategic and institutional approaches. Academy of Management Review, 20, 571-610.

VREDENBURG, H.; WESTLEY, F., 1993. Environmental leadership in three contexts: Managing for global competitiveness. Proceedings of the International Association of Business and Society, 495-500.

WINN, M., 1995. Corporate leadership and policies for the natural environment. En D. Collins y M. Stank (Eds.), Research in corporate social perform once and policy, supplement 1, JAI Press. Greenwich, CT., 127-161.

WINN, S. F.; ROOME, N. J., 1993. RyD management responses to environment: current theory and implications to practice and research. RyD Management, 23 (2), 147-160.

WOOD, D. J., 1991. Corporate social performance revisited. Academy of Management Review, 16, 691-718.

ZUCKER, L. G., 1987. Institutional theories of organization. En W. R. SCOTT y J. F. ShorT, Jr. (Eds.), Annual Review of Sociology, 13, Palo Alto, CA: 443-464 